\title{
Protocol Sequences for Multiple-Packet Reception: Throughput Invariance and User Irrepressibility
}

\author{
Yijin Zhang*, Yuan-Hsun Lo ${ }^{\dagger}$, Feng Shu* and Wing Shing Wong ${ }^{\ddagger}$ \\ ${ }^{*}$ School of Electronic and Optical Engineering \\ Nanjing University of Science and Technology \\ Email: yijin.zhang@gmail.com; shufeng@njust.edu.cn \\ ${ }^{\dagger}$ Department of Mathematics \\ National Taiwan Normal University \\ yhlo0830@gmail.com \\ $\ddagger$ Department of Information Engineering \\ The Chinese University of Hong Kong \\ wswong@ie.cuhk.edu.hk
}

\begin{abstract}
We consider the slot-synchronized collision channel without feedback, in which $K$ active users all transmit their packets to one sink. It is assumed that the channel has the ability of the multiple-packet reception (MPR), i.e., can accommodate at most $\gamma(1 \leq \gamma<K)$ simultaneous transmissions. Each user accesses the channel following a specific periodical zero-one pattern, called the protocol sequence, and transmits a packet if and only if the sequence value is equal to one. The fluctuation in throughput is incurred by random relative delay offsets among the beginning of protocol sequences due to the lack of feedback. There are two different design goals in the literature: throughput invariance and user irrepressibility. The former one enjoys constant throughput independent of relative delay offsets, and the latter one guarantees a non-zero individual throughput in the worst case. However, all previous performance evaluation on these two criteria assumed that $\gamma=1$. In this paper, we generalize these results to any $\gamma>1$. For both design objective, we establish a lower bound on sequence period and prove the lower bound can be achieved by some construction.
\end{abstract}

\section{INTRODUCTION}

\section{A. System Model}

As the model studied in this paper is based on the slot-synchronized collision channel without feedback (CCw/oFB) [1], we recall some of the basic concepts here. There are $K$ active users and one sink. We assume that all users are within the receiving range of the sink. The channel is time slotted. The packet length is fixed and occupies exactly a slot. Each user knows the slot boundaries and transmits its every packet within a channel slot, however, does not know the time offsets of the others. Generally, there are time delay offsets among these $K$ users. The channel access is done by assigning each user a deterministic binary sequence which is periodic. It is called protocol sequence [1]. The sequence of

This paper is a short version of the paper submitted to IEEE Trans. Inf. Theory, titled "Protocol Sequences for Multiple-Packet Reception", which is available at http://arxiv.org/abs/1401.6260.

This work was partially supported by the National Natural Science Foundation of China (No. 61301107, 61174060 and 61271230) and the Shenzhen Science and Technology Grant JCYJ20130401172046453. W. S. Wong is also a member of The Chinese University of Hong Kong Shenzhen Research Institute. user $i$ is $\mathbf{s}_{i}:=\left[s_{i}(0) s_{i}(1) \ldots s_{i}(L-1)\right]$, where $L$ is the common sequence period of all $K$ sequences. Also it has a relative shift $\tau_{i}$, which is a random integer measured in the unit of slot duration. User $i$ transmits a packet at slot $t$ if $s_{i}\left(t+\tau_{i}\right)=1$, and keeps silent if $s_{i}\left(t+\tau_{i}\right)=0$. The addition by $\tau_{i}$ is in modulo $L$. A packet can be received correctly if no other packets are being transmitted at the same slot. If two or more users transmit simultaneously, a collision occurs.

Recently, advanced reception techniques at the physical layer are employed to allow the channel to accommodate multiple communications. This has been referred to as the multiple-packet reception (MPR). Considering MPR, [2] studied ALOHA based random access, [3]-[6] proposed theoretical models and access protocols for IEEE 802.11, and [7], [8] further investigated the throughput and delay gains resulting from network coding [9]. In this paper, we assume that a packet can be received error-free if at most $\gamma-1(1 \leq \gamma<K)$ other packets are being transmitted simultaneously. Otherwise, any collided packet cannot be recovered. We refer to $\gamma$ as the MPR capability which was commonly assumed in [3]-[8]. The capacity of the collision channel without feedback under MPR is analyzed in [10], however, with the assumption that all collided packets have a fixed probability of being recovered, which is different from the MPR capability discussed here. To the authors' knowledge, there is no work on the behavior of protocol sequences considering the MPR capability $\gamma>1$.

We remark that there is no need of combining network coding and MPR in our system mode, since the sink is always available and only one-hop traffic is encountered.

\section{B. Design Criteria on Throughput}

The effective throughput of a user under the MPR capability $\gamma$ is defined as the fraction of time slots in which it send a packet without suffering any collision in which more than $\gamma$ users are involved. The variation in relative offsets among the users yields variation in throughput. As argued in [11], there are two extreme criteria in the design of protocol sequences: throughput invariance and user irrepressibility. 
The first criterion is to maximize the worst-case throughput, i.e., minimize the variation in throughput due to offsets. If the throughput of each user is constant and independent of any relative delay offsets, then the assigned protocol sequences are said to be throughput-invariant (TI). Shum et al. [12] proposed shift-invariant (SI) sequences which have perfect cross-correlation property, and proved that they are TI for $\gamma=1$. However, when $\gamma>1$, the impact of MPR capability on design of TI sequences is not examined. In this paper, we establish a lower bound on sequence period and prove that the construction of SI sequences [11] produces optimal TI sequences for any $\gamma$. Moreover, it is shown that the TI sequences must be SI in some cases.

The second design criterion is to guarantee that the throughput of each user is non-zero for any relative delay offsets. A user with such property can send at least one packet within the sequence period with probability one in the worst-case, in contrast to the random schemes. This strict guarantee is important in the application of monitoring medical activities and natural disasters. The protocol sequences with this performance are said to be user-irrepressible (UI). All protocol sequences in [11]-[16] can be found UI for $\gamma=1$. In order to minimize delay within which each user has transmitted a packet without collision, the objective of designing UI sequences is to make the period as short as possible. However, the minimum period of UI sequences has not been investigated for $\gamma>1$. We in this paper present the property of UI sequences for any $\gamma$ and give an asymptotically tight bound on sequence period for some cases by some known results from conflict-avoiding codes (CACs) [17]-[19].

Our work can be viewed as a generalization of results in [11], [12].

\section{Definitions And Notations}

We consider $K$ binary sequences $\mathbf{s}_{1}, \mathbf{s}_{2}, \ldots, \mathbf{s}_{K}$ of common period $L$. The offset of user $i$ is $\tau_{i}$ for $i=1,2, \ldots, K$.

The Hamming weight $w_{i}$ of $\mathbf{s}_{i}$ is defined as the number of ones in its sequence period, i.e., $w_{i}:=\sum_{t=0}^{L-1} s_{i}(t)$. The duty factor $f_{i}$ of $\mathbf{s}_{i}$ is defined as the fraction of ones of $\mathbf{s}_{i}$ in a period, for $i=1,2, \ldots, K$, i.e., $f_{i}:=\frac{1}{L} \sum_{t=0}^{L-1} s_{i}(t)$.

The cyclic shift of $\mathbf{s}$ by $\tau$ is denoted by

$$
\mathbf{s}^{(\tau)}:=[s(\tau) s(1+\tau) \ldots s(L-1+\tau)] .
$$

The throughput of user $i$ is defined as

$R_{i}\left(\tau_{1}, \tau_{2}, \ldots, \tau_{K}\right)=\frac{1}{L} \sum_{\substack{b_{i}=1, q \leq \gamma-1}} N\left(b_{1}, \ldots, b_{K} \mid \mathbf{s}_{1}^{\left(\tau_{1}\right)}, \ldots, \mathbf{s}_{K}^{\left(\tau_{K}\right)}\right)$,

in which $b_{i} \in\{0,1\}$ for all $i, q=\sum_{j \neq i} b_{j}$ and $N\left(b_{1}, \ldots, b_{K} \mid \mathbf{s}_{1}^{\left(\tau_{1}\right)}, \ldots, \mathbf{s}_{K}^{\left(\tau_{K}\right)}\right)$ denotes the number of time indices $t, 0 \leq t<L$, such that $s_{i}\left(t+\tau_{i}\right)=b_{i}$ for all $i$. This computes the fraction of time slots in which at most $\gamma$ users including user $i$ are transmitting. For simplicity, we sometimes use $R_{i}$ to denote the throughput of user $i$. To avoid the uninteresting cases, we only consider TI sequences with $R_{i}>0$ for any $i$ and any relative shifts.
We identify the $K$ users with $\mathcal{K}:=\{1,2, \ldots, K\}$. Let $\mathcal{O}_{K}$ be the set

$$
\bigcup_{n=1}^{K}\left\{\left(i_{1}, \ldots, i_{n}\right) \in \mathcal{K}^{n}: i_{1}<i_{2}<\cdots<i_{n}\right\}
$$

An element in $\mathcal{O}_{K}$ corresponds to an ordered tuple of users. For $\mathrm{A}=\left(i_{1}, i_{2}, \ldots, i_{n}\right) \in \mathcal{O}_{K}$, the generalized Hamming crosscorrelation associated with $\mathrm{A}$ is defined as

$$
H\left(\tau_{i_{1}}, \ldots, \tau_{i_{n}} ; \mathrm{A}\right):=\sum_{t=0}^{L-1} \prod_{j=1}^{n} s_{i_{j}}\left(t+\tau_{i_{j}}\right)
$$

Given an ordered tuple $\mathrm{A} \in \mathcal{O}_{K}$, then $H\left(\tau_{i_{1}}, \ldots, \tau_{i_{n}} ; \mathrm{A}\right)$ is said to be $\mathrm{SI}$ if it is equal to a constant for any $\tau_{i_{1}}, \ldots, \tau_{i_{n}}$.

A sequence set is said to be SI [12] if $H\left(\tau_{i_{1}}, \ldots, \tau_{i_{n}}\right.$; A $)$ is SI for every $\mathrm{A}$ in $\mathcal{O}_{K}$. A sequence set is said to be pairwise SI [15] if $H\left(\tau_{i_{1}}, \ldots, \tau_{i_{n}} ; \mathrm{A}\right)$ is SI for every $\mathrm{A}$ in $\mathcal{O}_{K}$ with $|A|=2$.

\section{TI SEQUENCES FOR MPR}

\section{A. Zero-variance Throughput}

In this subsection, we generalize the result in [12, Thm. 3] for $\gamma=1$ to those for $1 \leq \gamma<K$.

Lemma 1 ( [12, Lemma 2]). Suppose that $\mathbf{s}_{1}, \mathbf{s}_{2}, \ldots, \mathbf{s}_{n}$ are $n$ binary sequences of common period $L$. For $b_{1}, \ldots, b_{n} \in$ $\{0,1\}$, we have

$$
\sum_{\tau_{1}=0}^{L-1} \ldots \sum_{\tau_{n}=0}^{L-1} N\left(b_{1}, \ldots, b_{n} \mid \mathbf{s}_{1}^{\left(\tau_{1}\right)}, \ldots, \mathbf{s}_{n}^{\left(\tau_{n}\right)}\right)=L \prod_{i=1}^{n} N\left(b_{i} \mid \mathbf{s}_{i}\right) .
$$

Theorem 2. Let $\mathbf{s}_{1}, \mathbf{s}_{2}, \ldots, \mathbf{s}_{K}$ be $K$ TI protocol sequences for the MPR capability $\gamma(1 \leq \gamma<K)$ with duty factors $f_{1}, f_{2}, \ldots, f_{K}$ respectively. Then we have

$$
R_{i}=f_{i} \sum_{\substack{\mathrm{H} \subseteq \mathcal{K} \backslash\{i\} \\|\mathcal{H}|<\gamma}} \prod_{j \in \mathrm{H}} f_{j} \prod_{k \in \mathcal{K} \backslash(\{i\} \cup \mathrm{H})}\left(1-f_{k}\right) .
$$

Proof: Since TI protocol sequences produce zero-variance throughput, $R_{i}$ is a constant function of $\tau_{1}, \ldots, \tau_{K}$ and it must be equal to its average value. For purposes only of our proof, we now specify $\tau_{1}, \tau_{2}, \ldots, \tau_{K}$ are independent and uniformly distributed random variables that are equally likely to take on any of $L$ values: $0,1, \ldots, L-1$. After taking the expectation over $\left(\tau_{1}, \tau_{2}, \ldots, \tau_{K}\right)$, we obtain the average throughput of user 


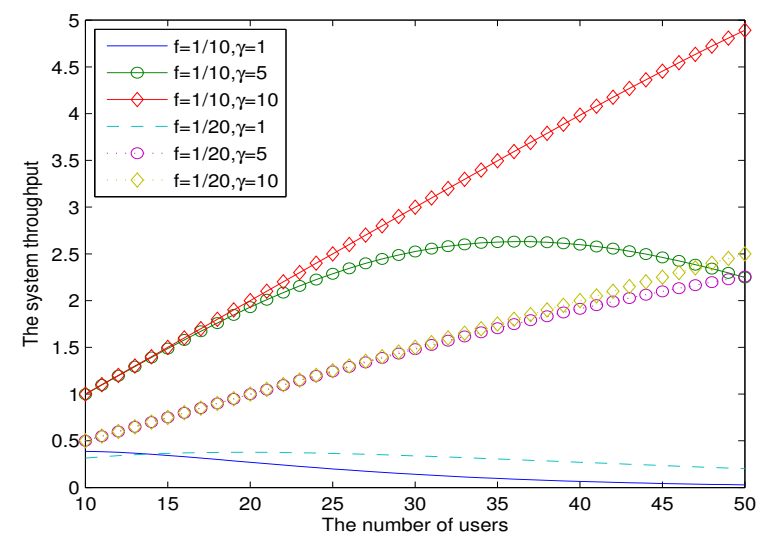

Fig. 1. The symmetric system throughput of TI sequences for $10 \leq K \leq 50$.

$i$ as the following:

$$
\begin{aligned}
\mathbb{E} & \left(R_{i}\left(\tau_{1}, \tau_{2}, \ldots, \tau_{K}\right)\right) \\
& =\frac{1}{L} \mathbb{E}\left(\sum_{q \leq \gamma-1, b_{i}=1} N\left(b_{1}, \ldots, b_{K} \mid \mathbf{s}_{1}^{\left(\tau_{1}\right)}, \ldots, \mathbf{s}_{K}^{\left(\tau_{K}\right)}\right)\right) \\
& =\frac{1}{L} \sum_{q \leq \gamma-1, b_{i}=1} \mathbb{E}\left(N\left(b_{1}, \ldots, b_{K} \mid \mathbf{s}_{1}^{\left(\tau_{1}\right)}, \ldots, \mathbf{s}_{K}^{\left(\tau_{K}\right)}\right)\right) \\
& =\frac{1}{L} \sum_{q \leq \gamma-1, b_{i}=1} \frac{1}{L^{K-1}} \prod_{j=1}^{K} N\left(b_{j} \mid \mathbf{s}_{j}\right) \\
& =\frac{1}{L^{K-1}} \sum_{q \leq \gamma-1} \frac{N\left(1 \mid \mathbf{s}_{i}\right)}{L} \prod_{j=1, j \neq i}^{K} N\left(b_{j} \mid \mathbf{s}_{j}\right) \\
& =\frac{1}{L^{K-1}} f_{i} \sum_{q \leq \gamma-1} \prod_{j=1, j \neq i}^{K} N\left(b_{j} \mid \mathbf{s}_{j}\right) \\
& =f_{i} \sum_{\mathrm{H} \subseteq \mathcal{K} \backslash\{i\}} \prod_{j \in \mathrm{H} \mid<\gamma} f_{j} \prod_{k \in \mathcal{K} \backslash(\{i\} \cup \mathrm{H})}\left(1-f_{k}\right),
\end{aligned}
$$

The equation (4) directly follows from the definition of the throughput of user $i$. The equation (5) is due to Lemma 1.

For the symmetric case that each user has the same duty factor $f$, each user enjoys the zero-variance throughput:

$$
\sum_{j=0}^{\gamma-1}\left(\begin{array}{c}
K-1 \\
j
\end{array}\right) f^{j+1}(1-f)^{K-1-j} .
$$

The results are shown in Fig. 1 for $10 \leq K \leq 50$ with $\gamma=$ $1,5,10$ and $f=1 / 10,1 / 20$, respectively.

\section{B. Lower bound on Minimum Period}

Long sequence period would bring instability of throughput on a short-time scale. We thus derive lower bounds on the period of TI protocol sequences for any $\gamma$. Let $\operatorname{gcd}(x, y)$ denote the greatest common divisor of $x$ and $y$.

We have a general definition on a given set of sequences.

Given a set of $K$ binary sequences, we divide it into two parts: $\mathcal{G}_{1}=\left\{\mathbf{s}_{1}, \ldots, \mathbf{s}_{M}\right\}$ and $\mathcal{G}_{2}=\left\{\mathbf{s}_{M+1}, \ldots, \mathbf{s}_{K}\right\}$ for some $M<K$. For $i=0,1, \ldots, M$ let $\lambda_{i}\left(\tau_{1}, \ldots, \tau_{M}\right)$ denote the number of time indices $t$ such that there are exactly $i$ ' 1 's among $s_{1}\left(t+\tau_{1}\right), \ldots, s_{M}\left(t+\tau_{M}\right)$. Similarly, for $i=M+1, \ldots, K$ let $\theta_{i}\left(\tau_{M+1}, \ldots, \tau_{K}\right)$ denote the number of time indices $t$ such that there are exactly $i$ ' 1 's among $s_{M+1}\left(t+\tau_{M+1}\right), \ldots, s_{K}\left(t+\tau_{K}\right)$. Define

$$
\theta_{\leq i}\left(\tau_{M+1}, \ldots, \tau_{K}\right)=\sum_{j=0}^{i} \theta_{j}\left(\tau_{M+1}, \ldots, \tau_{K}\right) .
$$

Note that $\lambda_{M}\left(\tau_{1}, \ldots, \tau_{M}\right)=H\left(\tau_{1}, \ldots, \tau_{M} ;(1, \ldots, M)\right)$.

Theorem 3. Let $\gamma$ be any integer such that $1 \leq \gamma<K$. If a set of $K$ binary sequences is TI for the MPR capability $\gamma$, then these $K$ sequences are pairwise $S I$.

Proof: Let $\mathcal{G}_{1}=\left\{\mathbf{s}_{1}, \mathbf{s}_{2}\right\}$ and $\mathcal{G}_{2}=\left\{\mathbf{s}_{3}, \mathbf{s}_{4}, \ldots, \mathbf{s}_{K}\right\}$ following the notations we defined at the beginning of this subsection. Then we choose the relative shifts $\tau_{3}^{*}, \tau_{4}^{*}, \ldots, \tau_{K}^{*}$ such that $\theta_{\gamma-1}\left(\tau_{3}^{*}, \ldots, \tau_{K}^{*}\right)>0$. This combination of relative shifts can always been found since there are at most $\gamma-1$ allone sequences in $\mathcal{G}_{2}$ for the case $1 \leq \gamma<K$. (If there are $\gamma$ all-one sequences, then all packets from other $K-\gamma$ sequences cannot be received error-free.) More precisely, assume in $\mathcal{G}_{2}$ that there are exactly $h$ all-one sequences. Then we cyclic shift some $\gamma-h-1$ non all-one sequences such that the first time slot of them are all equal to one, and cyclic shift the remaining sequences such that the first time slot of them are all equal to zero. Thus one sees there are exactly $\gamma-1$ ones in the first time slot.

Define $T_{1}$ as:

$$
T_{1}:=\sum_{\substack{b_{1}+b_{2}=1 \\ b_{3}+\ldots+b_{K}<\gamma}} N\left(b_{1}, \ldots, b_{K} \mid \mathbf{s}_{1}^{\left(\tau_{1}\right)}, \ldots, \mathbf{s}_{K}^{\left(\tau_{K}\right)}\right)
$$

and $T_{2}$ as:

$$
T_{2}:=\sum_{\substack{b_{1}+b_{2}=2 \\ b_{3}+\ldots+b_{K}<\gamma-1}} N\left(b_{1}, \ldots, b_{K} \mid \mathbf{s}_{1}^{\left(\tau_{1}\right)}, \ldots, \mathbf{s}_{K}^{\left(\tau_{K}\right)}\right) .
$$

We assume the relative shift between $\mathcal{G}_{1}$ and $\mathcal{G}_{2}$ is uniformly distributed in $0,1, \ldots, L-1$. After taking the expectation over this relative shift, by the elementary property of Hamming crosscorrelation, we have the following:

$$
\begin{aligned}
\mathbb{E}\left(L R_{1}+L R_{2}\right) & =\mathbb{E}\left(T_{1}+2 T_{2}\right)=\mathbb{E}\left(T_{1}\right)+2 \mathbb{E}\left(T_{2}\right) \\
& =\frac{1}{L} \sum_{i=1}^{2} i \lambda_{i}\left(\tau_{1}, \tau_{2}\right) \theta_{\leq \gamma-i}\left(\tau_{3}^{*}, \ldots, \tau_{K}^{*}\right) .
\end{aligned}
$$

Now we change the pair of relative shifts from $\left(\tau_{1}, \tau_{2}\right)$ to $\left(\tau_{1}^{\prime}, \tau_{2}^{\prime}\right)$. Let

$$
\sigma:=\lambda_{2}\left(\tau_{1}^{\prime}, \tau_{2}^{\prime}\right)-\lambda_{2}\left(\tau_{1}, \tau_{2}\right)
$$

By the fact that

$\lambda_{1}\left(\tau_{1}, \tau_{2}\right)+2 \lambda_{2}\left(\tau_{1}, \tau_{2}\right)=L f_{1}+L f_{2}=\lambda_{1}\left(\tau_{1}^{\prime}, \tau_{2}^{\prime}\right)+2 \lambda_{2}\left(\tau_{1}^{\prime}, \tau_{2}^{\prime}\right)$, we have

$$
\lambda_{1}\left(\tau_{1}^{\prime}, \tau_{2}^{\prime}\right)-\lambda_{1}\left(\tau_{1}, \tau_{2}\right)=-2 \sigma .
$$


Since $R_{1}+R_{2}$ is zero-variance, by (7) and (8), we have

$$
\begin{aligned}
& \sum_{i=1}^{2} i \lambda_{i}\left(\tau_{1}, \tau_{2}\right) \theta_{\leq \gamma-i}\left(\tau_{3}^{*}, \ldots, \tau_{K}^{*}\right) \\
& \quad=\sum_{i=1}^{2} i \lambda_{i}\left(\tau_{1}^{\prime}, \tau_{2}^{\prime}\right) \theta_{\leq \gamma-i}\left(\tau_{3}^{*}, \ldots, \tau_{K}^{*}\right) .
\end{aligned}
$$

Then from (9) and (10) we further obtain

$$
\sigma \theta_{\gamma-1}\left(\tau_{3}^{*}, \ldots, \tau_{K}^{*}\right)=0
$$

which implies that $\sigma=0$ because of the choice of $\tau_{3}^{*}, \ldots, \tau_{K}^{*}$. Thus, $\lambda_{2}\left(\tau_{1}, \tau_{2}\right)$ is a constant function of $\tau_{1}, \tau_{2}$.

Since $\lambda_{2}\left(\tau_{1}, \tau_{2}\right)=H\left(\tau_{1}, \tau_{2} ;(1,2)\right)$ and the choice of $\mathbf{s}_{1}, \mathbf{s}_{2}$ is arbitrary, we conclude that these $K$ sequences are pairwise SI for any $\gamma<K$.

Theorem 4 ( [15, Thm. 1]). The common period of any set of $K$ pairwise SI sequences with duty factors $n_{i} / d_{i}$, where $\operatorname{gcd}\left(n_{i}, d_{i}\right)=1$ for all $i$, is divisible by $d_{1} d_{2} \cdots d_{K}$. In particular, the minimum common period is at least $d_{1} d_{2} \cdots d_{K}$.

Theorem 5. Let $\gamma$ be an integer with $1 \leq \gamma<K$. If a set of $K$ binary sequences with duty factors $n_{i} / d_{i}$, where $\operatorname{gcd}\left(n_{i}, d_{i}\right)=$ 1 for all $i$, is TI for the MPR capability $\gamma$, then the period $L$ is divisible by $d_{1} d_{2} \cdots d_{K}$. In particular, the minimum common period is at least $d_{1} d_{2} \cdots d_{K}$.

Proof: It directly follows from Theorem 3 and Theorem 4.

\section{Optimal Construction}

Shum et al. [12] showed that any SI sequence set is TI for the ordinary model (i.e., $\gamma=1$ ). We extend this property to general $\gamma$ by means of the following result.

Theorem 6 ( [12, Thm. 1]). The sequence set $\mathbf{s}_{1}, \mathbf{s}_{2}, \ldots, \mathbf{s}_{K}$ is SI if and only if for each choice of $b_{1}, \ldots, b_{K}$, $N\left(b_{1}, \ldots, b_{K} \mid \mathbf{s}_{1}^{\left(\tau_{1}\right)}, \ldots, \mathbf{s}_{K}^{\left(\tau_{K}\right)}\right)$ is a constant function of $\tau_{1}, \ldots, \tau_{K}$.

Theorem 7. If a sequence set is SI, then it is TI for any MPR capability $\gamma$.

Proof: From (1), we obtain that the throughput $R_{i}\left(\tau_{1}, \tau_{2}, \ldots, \tau_{K}\right)$ can be computed only in terms of $N\left(b_{1}, \ldots, b_{K} \mid \mathbf{s}_{1}^{\left(\tau_{1}\right)}, \ldots, \mathbf{s}_{K}^{\left(\tau_{K}\right)}\right)$ for some particular choices of $b_{1}, \ldots, b_{K}$. By Theorem 6 , we find each term of the above is a constant function of $\tau_{1}, \ldots, \tau_{K}$ if the sequence set is SI. Thus $R_{i}\left(\tau_{1}, \tau_{2}, \ldots, \tau_{K}\right)$ is also a constant function of $\tau_{1}, \ldots, \tau_{K}$, which implies that a SI sequence set must be TI for any $\gamma$.

With the duty factors $n_{i} / d_{i}$, where $\operatorname{gcd}\left(n_{i}, d_{i}\right)=1$ for all $i$, the construction of SI sequences in [12] has the common period $d_{1} d_{2} \cdots d_{K}$. An interesting fact is that this construction indeed produces optimal TI sequences for any $1 \leq \gamma<K$ in the sense that the period achieves the lower bound in Theorem 5.
Example 1: The following are three sequences of period 27 with duty factors $2 / 3,1 / 3$ and $1 / 3$ :

$\mathbf{s}_{1}=\left[\begin{array}{lllllllllllllllllllllllllll}1 & 1 & 1 & 1 & 1 & 1 & 0 & 0 & 0 & 1 & 1 & 1 & 1 & 1 & 1 & 0 & 0 & 0 & 1 & 1 & 1 & 1 & 1 & 1 & 0 & 0 & 0\end{array}\right]$ $\mathbf{s}_{2}=\left[\begin{array}{lllllllllllllllllllllllllll}1 & 0 & 0 & 1 & 0 & 0 & 1 & 0 & 0 & 1 & 0 & 0 & 1 & 0 & 0 & 1 & 0 & 0 & 1 & 0 & 0 & 1 & 0 & 0 & 1 & 0 & 0\end{array}\right]$ $\mathbf{s}_{3}=\left[\begin{array}{lllllllllllllllllllllllllll}1 & 1 & 1 & 1 & 1 & 1 & 1 & 1 & 1 & 0 & 0 & 0 & 0 & 0 & 0 & 0 & 0 & 0 & 0 & 0 & 0 & 0 & 0 & 0 & 0 & 0 & 0\end{array}\right]$

One can check the above sequence set is SI and thus TI for any $\gamma$. We have $R_{1}=8 / 27, R_{2}=R_{3}=1 / 27$ for $\gamma=1$ and $R_{1}=16 / 27, R_{2}=R_{3}=7 / 27$ for $\gamma=2$ which are both in accordance with Theorem 2 . Its period is 27 and achieves the lower bound presented in Theorem 5 for $\gamma=1,2$.

\section{Structural Theorem}

The SI structure is necessary for optimal TI sequences under some technical conditions.

Theorem 8 ( [15, Thm. 3]). Let $p$ be a prime. If $K$ pairwise SI protocol sequences with duty factors $n_{i} / p$ for $i=1,2, \ldots, K$, have a common minimum period $p^{K}$, then they are SI.

Theorem 9. Let $p$ be a prime and $\gamma$ be a positive integer smaller than $K$. If a set of $K$ binary sequences with duty factors $n_{i} / p$ for $i=1,2, \ldots, K$ and period $p^{K}$, is $T I$ for the MPR capability $\gamma$, then these $K$ sequences are SI.

Proof: Directly follows from Theorem 3 and Theorem 8.

\section{UI SEQUENCES FOR MPR}

The following lemma provides a lower bound on the Hamming weight of any sequence in a UI sequence set for the MPR capability $\gamma$. The proof is straightforward and omitted.

Lemma 10. Let $\mathbf{s}_{1}, \mathbf{s}_{2}, \ldots, \mathbf{s}_{K}$ be $K$ UI sequences for the MPR capability $\gamma$. Then we have

$$
w_{i} \geq\left\lfloor\frac{K-1}{\gamma}\right\rfloor+1,
$$

for $i=1,2, \ldots, K$.

In order to investigate the most energy-efficient UI sequences, we in this section focus on the situation where each user transmits exactly $\lfloor(K-1) / \gamma\rfloor+1$ packets in a period, especially when $\gamma \mid K$. Note that the UI sequences with common Hamming weight $K$ for $\gamma=1$ studied in [11] is a special case here.

A set of binary sequences, say $\left\{\mathbf{s}_{1}, \mathbf{s}_{2}, \ldots, \mathbf{s}_{K}\right\}$, with common period is called a CAC with weight $w$ if each $w_{i}=w$ and $H\left(\tau_{i_{1}}, \tau_{i_{2}} ; \mathrm{A}\right) \leq 1$ for any $\mathrm{A}=\left(i_{1}, i_{2}\right) \in \mathcal{O}_{K}$ and any $\tau_{i_{1}}, \tau_{i_{2}}$. It is obvious that a CAC with weight $w$ and $K$ codewords provides a UI sequence set for

$$
\gamma \geq\left\lfloor\frac{K-1}{w}\right\rfloor+1
$$

In the case of $\gamma \mid K$, we have the following observation.

Proposition 11. Let $K=w \gamma$. A set of $K$ UI sequences of common Hamming weight $w$ for the MPR capability $\gamma$ is equivalent to a CAC with weight $w$ and $K$ codewords. 
Proof: Only the sufficient part needs to be verified. Since there is no all-zero sequence, the pairwise Hamming crosscorrelation is always non-zero. Assume by contradiction that $H\left(\tau_{1}, \tau_{2} ;(1,2)\right) \geq 2$. Then, one can adjust $\tau_{3}, \tau_{4}, \ldots, \tau_{K}$ in such a way that each one in $\mathbf{s}_{1}^{\left(\tau_{1}\right)}$ is blocked by at least $\gamma$ transmissions, which contradicts to the UI property.

Example 2: The following six sequences of period 25 form a CAC with weight 3 , and thus a UI sequence set for $\gamma=2$.

$$
\begin{aligned}
& \mathbf{s}_{1}=\left[\begin{array}{lllllllllllllllllllllllll}
1 & 1 & 1 & 0 & 0 & 0 & 0 & 0 & 0 & 0 & 0 & 0 & 0 & 0 & 0 & 0 & 0 & 0 & 0 & 0 & 0 & 0 & 0 & 0 & 0
\end{array}\right] \\
& \mathbf{s}_{2}=\left[\begin{array}{lllllllllllllllllllllllll}
1 & 0 & 0 & 0 & 1 & 0 & 0 & 0 & 1 & 0 & 0 & 0 & 0 & 0 & 0 & 0 & 0 & 0 & 0 & 0 & 0 & 0 & 0 & 0 & 0
\end{array}\right] \\
& \mathbf{s}_{3}=\left[\begin{array}{lllllllllllllllllllllllll}
1 & 0 & 0 & 0 & 0 & 0 & 0 & 0 & 0 & 1 & 0 & 0 & 0 & 0 & 0 & 0 & 0 & 0 & 1 & 0 & 0 & 0 & 0 & 0 & 0
\end{array}\right] \\
& \mathbf{s}_{4}=\left[\begin{array}{lllllllllllllllllllllllll}
1 & 0 & 0 & 0 & 0 & 0 & 0 & 0 & 0 & 0 & 0 & 1 & 0 & 0 & 0 & 0 & 0 & 0 & 0 & 0 & 0 & 0 & 1 & 0 & 0
\end{array}\right] \\
& \mathbf{s}_{5}=\left[\begin{array}{lllllllllllllllllllllllll}
1 & 0 & 0 & 0 & 0 & 0 & 1 & 0 & 0 & 0 & 0 & 0 & 1 & 0 & 0 & 0 & 0 & 0 & 0 & 0 & 0 & 0 & 0 & 0 & 0
\end{array}\right] \\
& \mathbf{s}_{6}=\left[\begin{array}{lllllllllllllllllllllllll}
1 & 0 & 0 & 0 & 0 & 1 & 0 & 0 & 0 & 0 & 1 & 0 & 0 & 0 & 0 & 0 & 0 & 0 & 0 & 0 & 0 & 0 & 0 & 0 & 0
\end{array}\right]
\end{aligned}
$$

Among all CACs of period $L$ and weight $w$, let $M(L, w)$ denote the maximum number of codewords. The following asymptotic bound was shown in [18]:

$$
\limsup _{L \rightarrow \infty} \frac{M(L, w)}{L}=\frac{1}{2 w-2} .
$$

On the other hand, let $L(K, w)$ be the smallest period $L$ such that a CAC with weight $w$ and $K$ codewords exists. By definition, we have

$$
\limsup _{K \rightarrow \infty} \frac{K}{L(K, w)}=\limsup _{L \rightarrow \infty} \frac{M(L, w)}{L}
$$

and thus

$$
\limsup _{K \rightarrow \infty} \frac{2 w K}{L(K, w)}=1 \text {. }
$$

Given positive integers $\gamma$ and $K$ with $\gamma \mid K$, let $L_{\gamma}(K)$ denote the smallest period $L$ such that a set of $K$ UI sequences of weight $K / \gamma$ for MPR capability $\gamma$ exists. By Proposition 11 , one has $L_{\gamma}(K)=L(K, K / \gamma)$. Hence, from (12), we have the following result, which is an extension of [11, Theorem 7]. Note that the involved sequence is of positive rational numbers.

\section{Theorem 12.}

$$
\begin{gathered}
\liminf _{K \rightarrow \infty} \frac{L_{\gamma}(K)}{2 K^{2} / \gamma}=1 . \\
\text { V. CONCLUSION }
\end{gathered}
$$

\section{CONClusion}

In this paper, we investigate a collision MPR channel without feedback. We generalize the results of [11], [12], i.e., study TI and UI sequences for any MPR capability $\gamma$. We prove that TI sequences must be pairwise SI for any $\gamma$ and the period thus grows like $K^{K}$, the construction of SI sequences in [12] indeed produces optimal TI sequences for any $\gamma$, and the SI property is intrinsic for optimal TI sequences if the duty factor meets some technical conditions. On the other hand, we find that the UI sequences studied in this paper are equivalent to a CAC with the weight $K / \gamma$ and $K$ codewords. It is further shown that an asymptotically optimal construction have the sequence period approximately $2 K^{2} / \gamma$.
For ad hoc network or multi-hop traffic, one would note that network coding can significantly increase the performance of access protocol with MPR, as presented in [7], [8]. Our followup work seeks to explore the sequence design simultaneously considering network coding and MPR.

\section{REFERENCES}

[1] J. L. Massey and P. Mathys, "The collision channel without feedback," IEEE Trans. Inform. Theory, vol. 31, no. 2, pp. 192-204, Mar. 1985.

[2] V. Naware, G. Mergen, and L. Tong, "Stability and delay of finite user slotted ALOHA with multipacket reception," IEEE Trans. Inform. Theory, vol. 51, no. 7, pp. 2636-2656, 2005.

[3] Y. Zhang, P. Zheng, and S. Liew, "How does multiple-packet reception capability scale the performance of wireless local area networks?" IEEE Transactions on Mobile Computing, vol. 8, no. 7, pp. 923-935, Jul. 2009.

[4] F. Babich and M. Comisso, "Theoretical analysis of asynchronous multi-packet reception in 802.11 networks," IEEE Transactions on Communications, vol. 58, no. 6, pp. 1782-1794, Jun. 2010.

[5] D. Chan, T. Berger, and L. Tong, "Carrier sense multiple access communications on multipacket reception channels: Theory and applications to IEEE 802.11 wireless networks," IEEE Transactions on Communications, vol. 61, no. 1, pp. 266-278, Jan. 2013.

[6] A. Mukhopadhyay, N. Mehta, and V. Srinivasan, "Design and analysis of an acknowledgment-aware asynchronous MPR MAC protocol for distributed WLANs," IEEE Transactions on Wireless Communications, vol. 12, no. 5, pp. 2068-2079, May 2013.

[7] A. Rezaee, L. Zeger, and M. Medard, "Multi packet reception and network coding," in IEEE MILCOM 2010, San Jose, Oct. 2010, pp. 1393-1398.

[8] J. Cloud, L. Zeger, and M. Medard, "MAC centered cooperationsynergistic design of network coding, multi-packet reception, and improved fairness to increase network throughput," IEEE Journal on Selected Areas in Communications, vol. 30, no. 2, pp. 341-349, Feb. 2012.

[9] R. Ahlswede, N. Cai, S.-Y. Li, and R. Yeung, "Network information flow," IEEE Trans. Inform. Theory, vol. 46, no. 4, pp. 1204-1216, 2000.

[10] S. Tinguely, M. Rezaeian, and A. J. Grant, "The collision channel with recovery," IEEE Trans. Inform. Theory, vol. 51, no. 10, pp. 3631-3638, Oct. 2005 .

[11] K. W. Shum, W. S. Wong, C. W. Sung, and C. S. Chen, "Design and construction of protocol sequences: shift invariance and user irrepressibility," in IEEE Int. Symp. Inform. Theory, Seoul, Jun. 2009, pp. 13681372 .

[12] K. W. Shum, C. S. Chen, C. W. Sung, and W. S. Wong, "Shift-invariant protocol sequences for the collision channel without feedback," IEEE Trans. Inform. Theory, vol. 55, no. 7, pp. 3312-3322, Jul. 2009.

[13] N. Q. A, L. Györfi, and J. L. Massey, "Constructions of binary constantweight cyclic codes and cyclically permutable codes," IEEE Trans. Inform. Theory, vol. 38, no. 3, pp. 940-949, May 1992.

[14] W. S. Wong, "New protocol sequences for random access channels without feedback," IEEE Trans. Inform. Theory, vol. 53, no. 6, pp. 20602071, Jun. 2007.

[15] Y. Zhang, K. W. Shum, and W. S. Wong, "On pairwise shift-invariant protocol sequences," IEEE Commun. Lett., vol. 13, no. 6, pp. 453-455, Jun. 2009.

[16] K. W. Shum and W. S. Wong, "Construction and applications of CRT sequences," IEEE Trans. Inform. Theory, vol. 56, no. 11, pp. 5780-5795, Nov. 2010.

[17] K. Momihara, M. Müller, J. Satoh, and M. Jimbo, "Constant weight conflict-avoiding codes," SIAM J. Discrete Math., vol. 21, no. 4, pp. 959-979, 2007.

[18] K. W. Shum and W. S. Wong, "A tight asymptotic bound on the size of constant-weight conflict-avoiding codes," Des. Codes Cryptogr., vol. 57, no. 1 , pp. 1-14, Oct. 2010.

[19] K. W. Shum, W. S. Wong, and C. S. Chen, "A general upper bound on the size of constant-weight conflict-avoiding codes," IEEE Trans. Inform. Theory, vol. 56, no. 7, pp. 3265-3276, Jul. 2010. 